\title{
REPLACING TRADITIONAL TELEPHONY WITH VOIP
}

\author{
Collin Jackson, Texas A\&M University - Kingsville, collin_jackson@sbcglobal.net \\ Jack D. Shorter, Texas A\&M University - Kingsville, jack.shorter@tamuk.edu \\ Karen A. Forcht, North Carolina A\&T University, kaforcht@ ncat.edu
}

\begin{abstract}
Voice-over-Internet protocol (VoIP) is an emerging technology that is beginning to replace traditional telephone services because it is much more cost effective for the consumer and businesses. Long distance charges are relatively a thing of the past, and in general there are more feature-rich services available over a VoIP line than traditional phone services. VoIP provides phone services without utilizing the expensive infrastructure of the traditional telephone company. VoIP is an enhancement to our traditional ways of communicating via the telephone; users can combine voice and video in a single call, share data, and accomplish many other tasks that are impossible over traditional phone lines. Businesses can utilize Session Initiated Protocol (SIP) over VoIP to eliminate the need for large PTSN trunk lines and wasted space. VoIP is the future and its features will eventually far exceed traditional landline phone services. But with any new technology there are also disadvantages in utilizing leading edge services such as VoIP. Some disadvantages include dropped calls, poor voice quality, or even cost a business more than traditional systems in regards to outages and lost production time. If the Internet connection goes down, so does your phone access. Access to 911 emergency services may not function correctly at critical times.
\end{abstract}

Keywords: VoIP, Voice-over-Internet Protocol.

\section{VOIP OVER GEOGRAPHICALLY SEPARATE REGIONS}

Many businesses across the globe are taking advantage of a unified communications system by deploying VoIP technologies throughout their company. Over the last few years the industry has been seeing this gradual separation between phone numbers and specific geographic regions [2]. As Voice-over-Internet Protocol grows, landline phone services will begin to wane; its outdated infrastructure being replaced by a virtual infrastructure spanning the globe utilizing the Internet. Because VoIP devices can be used any where an internet connection is available, your phone number is not tied to any particular region. This can come in extremely handy for business users who telecommute across great distances, and frequently travel in their business [9].

As companies embrace VoIP many are replacing traditional PBX systems with VoIP SoftPhones or VoIP PBX Systems using SIP. It appears that most companies that have made this switch have reported dramatic cost savings of up to $40 \%$ on domestic calls and $90 \%$ on international calls. The is due to the fact that they no longer have to rely on the costly ISDN primary rate interfaces, or dedicated gateways to the public switched telephone network (PTSN) [1].

VoIP services are continuing to advance and many cellular companies are beginning to adapt to this growing technology by developing cell phones that allow users to switch between a traditional cell tower and VoIP access when they are close enough to wireless hotspots [14]. This trend has lead to advances in wireless technologies for both data and voice communication applications paving the way for a new market of telecommunications products.

Because of this fast growing technology, we believe that VoIP is already a viable replacement to the existing wire line services and the trend is expanding to include cellular telephone communications and other forms of wireless communication. As more and more cities introduce wireless hotspots, it will not be long before VoIP from mobile devices is a viable replacement to traditional cellular communication devices [14]. Businesses who rely on call centers in various locations of the world are able to save money and cut costs on long distance charges and the amount of telephone hard lines (PTSN trunks). The problem of connecting a call originating from different carriers or countries through standard tollfree hotlines can be done without building a dedicated infrastructure over existing PTSN systems. These companies rely heavily on the vast amount of features and cost saving that combining voice and data on a single infrastructure can provide.

Any company would be negligent not to consider utilizing VoIP technologies in their business, and many businesses have realized that in the current economy every dollar saved will help the institution 
overcome some of the problems of the economic downturn.

\section{VoIP COST SAVINGS AND LONG DISTANCE}

Voice-over-Internet Protocol (VoIP) services include free long distance, or significantly lower cost long distance when calling internationally, compared to traditional PTSN services. This is due to the fact that VoIP services do not have to maintain the vast and expensive wire infrastructure that traditional telephone services are dependent on. VoIP services are able to provide this technology at much discounted prices. VoIP includes long distance at no additional charge. This makes it more economical to businesses and consumers who frequently make long distance calls or conduct business over great distances. Traditional phone service do not even compare in regards to long distance coverage. Customers can save over $\$ 500$ per year by using a VoIP service because long distance is included with the monthly fee. This is different than traditional phone service providers who charge both a monthly long distance service fees and usage minutes during the billing period [13].

Voice-over-Internet Protocol (VoIP) will eventually replace the existing PTSN services, and the internet technologies that allow VoIP to be successful will continue to improve and make the service even more desirable. Even though VoIP is still a fairly new technology, advances have been made which improve the quality of service bringing VoIP technology closer to replacing traditional PTSN services. As we have previously stated, everyone is familiar with the dreaded long distance bill. From corporate giants to small town businesses everyone has to deal with long distance charges. VoIP is slowly but surely causing the existing landline phone services to increase their prices without really increasing customer service or products.

Given the current state of the economy it is no surprise that VoIP has continued to grow at a steady rate. More and more companies are looking for ways to save money and conserve what income they can. Companies using VoIP will have additional cash to survive and they will be able to continue research and development of this communications platform [3]. VoIP is here to stay and the depressed economy has a huge role in its success. VoIP technology is gaining acceptance and becoming a close real competitor and alternative to traditional landline telephone companies. It should lead to a complete restructuring of the existing telecommunications industry. VoIP just has more features and cost savings opportunities than the current traditional communications vendors are able to offer.

\section{VOIP FEATURES EXCEED TRADITIONAL PHONE SERVICES}

VoIP offers feature rich services at lower costs and customers in Europe are flocking to this new service according to a recent study released by TeleGeography. In this study TeleGeography predicts that VoIP lines will spike from 35 million subscribers in Europe in 2008 to 48 million in 2009 [6], and in Hungary customers are fleeing from traditional land lines [7]. This is quite a surprising trend. European traditional telecommunications provider customers are finding a newer and better quality of service using VoIP. Traditional providers will have to adapt their infrastructure in order to contend with VoIP's growing feature list and market share.

VoIP service providers offer customers features such as caller id, call waiting, call transfer or the ability to transfer a call already in progress to another device, such as a cell phone. Other features include call forwarding, voicemail which can be checked in multiple places via email, or even from another phone with the correct password. Customers even have the ability to change their phone number themselves at no additional cost, add or remove additional phone lines or add virtual numbers in other area codes to their account [12]. These features are some that one would never be able to find on a traditional PTSN system, because the infrastructure does not allow for it, it was not designed to accommodate the vast amounts of information the Internet is capable of handling.

Because Voice-over-Internet Protocol (VoIP) does not have to maintain a vast wire line infrastructure customers are able to achieve lower rates, generally starting around $\$ 19.99$ a month. Using a traditional phone company even with the vast amount of features it comes with one would be paying over $\$ 80$ a month plus taxes, fees, and other charges. Businesses generally support and maintain large bundles of cables called trunks which are connected to a local private branch exchange (or PBX) system. Companies with a large amount of phone lines typically have hundreds of pairs of copper lines and the necessary equipment to connect these wires to their PBX system. There is a substantial cost for businesses using traditional phone services. If businesses switch to VoIP, they can eliminate this expense almost entirely, because hundreds of lines 
can be delivered over a single high-speed internet connection.

In addition to VoIP technology allowing users to replace hundreds of physical wires, VoIP can provide redundancy to your telecommunications infrastructure just by utilizing two or more separate high-speed internet connections. One would be able to access their services even if one connection was physically disconnected or damaged. In order to accomplish the same task with standard phone cabling methods, all of the cabling would have to be doubled to achieve reliable redundancy. Businesses that rely heavily on telecommunications would do well to convert to VoIP technologies in order to protect themselves from the potential of a serious disruption to their daily business due to line faults in their traditional PTSN system [12].

Why pay the phone company to terminate and install large and very bulky copper trunk lines when VoIP can bring your business as many phone numbers from any geographic location around the world? It is not practical for a business to purchase and amass the significant startup costs involved in traditional phone system installations with a more cost effective solution like VoIP or SIP trunking (Session Initiated Protocol, multiple VoIP lines through a single connection).

In the latest specifications a single 10 megabit symmetric connection can provide for over 48 completed concurrent calls through PBX systems utilizing the latest VoIP SIP trunking protocols and optional voice compression codec G.729. This latest advance in VoIP technology was announced by Speakeasy, who currently is certifying eight new IP based PBX vendors with the ADTRAN and Fonality being the first two approved [8].

Many businesses are beginning to combine voice, data, and video in a single cohesive system to enhance business communication and customer service. Users can utilize these combined systems for standard VoIP communication. They can also perform enhanced functions such as conference calling, video conferencing, and data sharing which highlights VoIP technology even further. VoIP and Session Initiated Protocol (SIP) are the wave of the future promising a much more flexible communications platform than current PTSN phone systems could ever hope to achieve without an undermined expense for a complete overhaul of the current infrastructure to accommodate these new services that VoIP and SIP are already able to provide.

Businesses that have unified communications throughout their company benefit the most from VoIP. In many cases it is not economical to upgrade an existing traditional system that is still working unless existing costs are the cause for major expenditures for upkeep in your current information technology budgets [5]. Another advantage of utilizing VoIP in the business environment is it is relatively easy to deploy. It is also easy to setup a new user extension, move an existing extension or change settings. To do this, a traditional phone system would require a dedicated connection back to the PBX [5]. A VoIP phone can be plugged into any ordinary network connection along side the user's computer, or in some cases it can be plugged directly into the user's computer. This means less physical infrastructure is required to deploy VoIP in the business, which can result in substantial cost savings.

Companies such as MagicJack and Skype have even created a VoIP system that allows users to utilize VoIP without having anything more than a computer and high-speed internet connection. This is simpler than a traditional phone company could provide. This feature of VoIP makes traveling and maintaining communication capabilities while abroad so much easier. Skype a European based company has a software based VoIP solution that allows users to communicate freely with other Skype users using voice, video, and even text messaging in a single easy to use application and MagicJack allows customers to use either a software interface or connect a standard telephone to the device and use their VoIP services from any computer that has a high-speed internet connection [10].

Software solutions to VoIP allow other forms of data communication to be integrated into a unified solution giving the user more intuitive options. Users are able to access their email, company directory, and share documents and other information among colleagues any where in the world. The capability for a VoIP to be integrated with other communications mediums is another advantage that VoIP has over traditional telephone communications. This technology can only support voice communication and limited data communication which first has to be converted to analog data signals. As VoIP continues to grow, users have even begun using fax machines over a VoIP connection. They have also intercepted inbound facsimile calls and read them directly into an email or other digital data 
media instead of actually producing the physical print copy. Fax over Internet Protocol (FoIP) is not a perfect technology and has even more disadvantages than VoIP connections because of the limited frequency range in the compression codecs used by VoIP and packet loss issues which can cause page loss or indescribable data in a faxed document. FoIP is not a subject of discussion for this paper, and was only included to point out some of the other uses of VoIP technologies, and other features of traditional phone services are capable of being replaced with VoIP systems.

\section{DISADVANTAGES OF VoIP}

Although there are several pitfalls to using VoIP services, one major pitfall is the problems with the 911 emergency services. Traditional phone systems are able to pass the call origin, location, customer information and other relevant data to the Public Safety Answering Point (PSAP) facility, but on a VoIP connection this capability may not work correctly causing delays in emergency situations. This is caused by the fact that VoIP allows users to separate their phone number from geographic ties that existing systems rely on. VoIP providers have had to work extra hard in order to provide properly functioning 911 services to their customers [4].

Usually call origin is determined based on the customer account information generally collected during account setup, however for the traveling user this information may frequently change, and VoIP providers have had to implement a means to allow customers to rapidly change and update this information quickly and efficiently regardless of where they happen to be using their service. The call must be routed to the proper PSAP facility according to FCC regulations [4]. As VoIP technology progresses, this pitfall may be remedied by relying on services already in development such as GeoIP facilities, which create and maintain lists of IP addresses and associated geographic locations. But many believe this would be a bit invasive to privacy and allow systems to be more susceptible to eavesdropping.

Occasionally during peak call periods or peak bandwidth usage, a disturbance in the connection known as jitter may occur. Jitter is caused because of serialization, packet queuing, transit delay, or either slow or heavily congested links, this problem may be alleviated but not fully resolved by implementing the use of Quality of Service (QoS) control mechanisms to classify bandwidth utilization for specific services and help alleviate bandwidth congestion. Because this pitfall is a problem with the architecture of IP networking and not specific to VoIP technologies, this problem may exist for sometime and not be fully remedied with current technologies [11].

Business users need to take into account that not all their calls will be of the same quality they are accustomed to when utilizing VoIP. This is the same situation private users experience because of network congestion or other factors affecting the network bandwidth capabilities. Because VoIP has disadvantages that traditional phone systems do not have it is important to examine the viability of VoIP and consider the communication needs of your business [5]. In businesses, communication plays a vital role in the survival of said business. Therefore, selecting a viable and stable communication system is necessary no matter which system you choose.

A company must analyze factors such as the number of remote workers; turnover rate, long distance costs, network stability and potential need for expansion accommodate VoIP. The necessity for a unified communications need must be addressed [5]. If a company is unable to find a reason within these factors, VoIP unfortunately is not an option for that company. According to Michael Lemm owner of FeedomFire Communications and Business-VoIPSolutions.com, VoIP is not completely unavailable to any business. There are other options such as PBX gateways that service traditional analog phone lines but allow for VoIP lines to be used for external numbers. This will allow many businesses to take advantage of the cost saving advantages for long distance service by using VoIP to complete those calls [5].

In large organizations it may be necessary to keep VoIP and standard data communications networks separate which can increase the cost of deployment of a VoIP infrastructure considerably, which may exceed the existing cost of current communication systems already in place within an organization. The initial cost of deployment may be a major negative for large enterprises because of VoIP phones for enterprise use can cost more than standard PBX phones and VoIP phones require more stringent network configurations in order to properly function and provide adequate service.

VoIP connections will prove to be more stable, but for now, users will have to manage the occasional jitter. One major disadvantage stems from the fact 
that VoIP data is sent over the internet and is susceptible to packet loss and drop outs entirely due to extreme packet loss or lack of bandwidth [4]. In some cases due to the architecture of some VoIP solutions, it may be required that that both parties be VoIP users, especially when dealing with Software VoIP solutions like Skype [4]. Many of these disadvantages will be all but eliminated in time as with any new technology it can only improve, but for now most customers are able to adequately work around these issues to make VoIP suit their needs.

Another problem is VoIP and SIP calls are not as secure as traditional telecommunication systems because they run on an IP network which can be intercepted anywhere on the internet in transit between the calls origin and call destination. This can be a major drawback which can disqualify the use of this technology especially for certain applications such as in the medical industry where laws such as HIPPA have strict procedures governing how medical records must be stored and secured. Some situations have been reported where basic VoIP features are billed "a la cart" and this can lead to even greater expenses as opposed to a traditional phone system [5].

\section{CONCLUSIONS}

Because VoIP is rapidly growing and replacing plain old telephone services, existing telephone services may become obsolete very soon. VoIP may moderate long distance charges. Customers will be able to answer their phone any where. Their phone number will not be tied to any geographic location. Even though VoIP still has disadvantages, they are not enough to dissuade people from utilizing this technology. The calls they are making are free or extremely low cost compared to using a traditional phone services.

VoIP and SIP solutions for business are providing a way to give users multiple services over a single cohesive architecture. By utilizing VoIP a company is able to save on the costly overhead expenses of maintaining multiple infrastructures to carry their voice, data, video, and other media throughout their organization. Consumers are able to benefit as well through cost savings because they do not have to pay for long distance, monthly service fees for basic features, and are able to manage most of their service capabilities without the need to call customer service.

Even with the disadvantages of VoIP when compared to traditional wire-line services, many customers have been completely satisfied with the service once they have become accustomed to the few and occasional problems one may experience on a VoIP connection. Many users take advantage of the unlimited calling capabilities, free long distance, easy to access voicemail, and many other features at costs that are considerably less than a traditional phone company's rates.

Because VoIP systems are relatively cheap and easier to deploy than traditional phone systems, we would say that it has revolutionized our communications capabilities and will be around for many years before something newer and better replaces it, or expands on VoIP. The Internet is constantly growing and expanding and VoIP technologies will have a place in the future of communications and specifically Internet communications.

\section{REFERENCES}

1. Brooks, Rosemary G. (2009, March 02). How PBX Replacement Systems and Softphones Can Help Your Business. EZine Articles, Retrieved March 04, 2009, from http://ezinearticles.com/?How-PBXReplacement-Systems-and-Softphones-Can$\underline{\text { Help-Your-Business\&id=2053116 }}$

2. Courtney, Jim (2008, November 12). Area Codes Are Dead - Thank VoIP. Retrieved March 04, 2009, from http://gigaom.com/2008/11/12/one-numberfor-worldwide-local-access-becoming-areality/

3. Ford, Carl (2009, February 10). Way to go Danny. FierceVoIP, Retrieved March 06, 2009, from http://www.fiercevoip.com/story/waygo-danny/2009-02-10

4. Jarred, Jeff (2009, February 17). Disadvantages of Voice Over IP. EZine Articles, Retrieved March 04, 2009, from http://ezinearticles.com/?Disadvantages-of$\underline{\text { Voice-Over-IP\&id=2005752 }}$

5. Lemm, Michael (2009, March 05). Pros and Cons of VoIP For Small Business - Is it Right For You Or Not?. Retrieved March 9, 2009, from EZine Web site: http://ezinearticles.com/?Pros-and-Cons-ofVoIP-For-Small-Business---Is-it-Right-For$\underline{\text { You-Or-Not?\&id=2069407 }}$ 
6. Mohney, Doug (2009, March 05). TeleGeography: 35M VoIP lines in Europe in 2008, will reach 48M in 2009. Retrieved March 06, 2009, from http://www.fiercevoip.com/story/telegeogra phy-voip-tops-35m-lines-2008-predicts48m-2009/2009-03-05

7. Mohney, Doug (2009, January 12). SPOTLIGHT: Landlines subs down in Hungary. Retrieved March 06, 2009, from http://www.fiercetelecom.com/story/spotligh t-landlines-subs-down-hungary/2009-01-12

8. Mohney, Doug (2009, March 03). Speakeasy gets SIP trunking, certifies ADTRAN, Fonality. FierceVoIP, Retrieved March 06, 2009, from http://www.fiercevoip.com/story/speakeasygets-sip-trunking-certifies-adtran-fonalityadds-voip-ethernet/2009-03-03

9. Unuth, Nadeem (2009). Reasons for Choosing Voice over IP - VoIP Advantages. Retrieved March 8, 2009, from About.Com Web site: http://voip.about.com/od/voipbasics/a/Reaso nsForVoIP.htm

10. Unuth, Nadeem (2009). Travel Globally. Retrieved March 8, 2009, from About.com Web site: http://voip.about.com/od/voipfeatures/g/trav elglobally.htm

11. VoIP Troubleshooter, LLC, Indepth: Jitter. Retrieved March 9, 2009, from VoIP Troubleshooter Web site: http://www.voiptroubleshooter.com/indepth/ jittersources.html

12. WhichVoIP (2009). WhichVoIP's Guide to VoIP Phone Service Features and Terms. Retrieved March 8, 2009, from WhichVoIP Web site: http://www.whichvoip.com/voip/info/voip_f eatures.htm

13. WhichVoIP (2009). Cheap Home Phone Service: Use VoIP for Cheap Home Phone Service Save Over \$500/Yr. Retrieved March 8, 2009, from WhichVoIP Web site: http://www.whichvoip.com/voip/voip_savin gs.htm
14. Xavier, M (2007, Dec 13). Raising the Bar Continuing Advances in Voip Technology. The Free Library, Retrieved March 06, 2009, from http://www.thefreelibrary.com/Raising+the+ Bar+Continuing+Advances+in+Voip+Techn ology-a01073816615 